\title{
不同风沙条件对几种植物生态生理特征的影响
}

\author{
于云江 ${ }^{12} \quad$ 史培军 $^{1}$ 鲁春霞 $^{3}$ 刘家琼 $^{2}$ \\ (1 北京师范大学资源研究所 环境演变与自然灾害教育部重点实验室, 北京 100875) \\ (2 中国科学院寒区早区环境与工程研究所沙坡头沙漠试验研究站, 兰州 730000) \\ (3 中国科学院地理科学与资源研究所, 北京 100101)
}

\begin{abstract}
摘 要 在野外风洞条件下 就不同风况的风沙胁迫对几种固沙植物生态生理特征的影响进行了实验研究。实验 采用了沙坡头地区的野外植物和盆栽植物。在不同风速 $\left(5.9 \mathrm{~m} \mathrm{~s}^{-1}, 7.9 \mathrm{~m} \mathrm{~s}^{-1}, 9.9 \mathrm{~m} \mathrm{~s}^{-1}, 14 \mathrm{~m} \mathrm{~s}^{-1}, 10 \mathrm{~m} \mathrm{~s}^{-1}\right.$, $\left.15 \mathrm{~m} \mathrm{~s}^{-1}, 20 \mathrm{~m} \mathrm{~s}^{-1}\right)$ 和吹袭时间间隔 $(2 \mathrm{~d}, 4 \mathrm{~d}, 9 \mathrm{~d})$ 下测定了植物 3 个生理生态参数的变化。实验结果表明 :1) 净 风和风沙流胁迫均可使植物的净光合速率 $(P n)$ 降低, 蒸腾速率 $(E)$ 升高, 从而导致水分利用效率 $(W U E)$ 下降。净 风和风沙流对植物生理生态特征的影响显著不同。同样为 $14 \mathrm{~m} \mathrm{~s}^{-1}$ 的风速时，风沙流使植物的 $P n$ 降低 $40.7 \%$, 而 净风使其降低了 $35.88 \%$ 。2)吹袭的时间间隔越短 植物的净光合速率降幅越大 ; 风沙流比净风的影响更大。在 2 $\mathrm{d}, 4 \mathrm{~d}, 9 \mathrm{~d}$ 为间隔的风沙流吹袭下, 油蒿 (Artemisia ordosica) 的 Pn 分别下降了 $20.13 \% 、 11.76 \% 、 7.72 \%$ 。风沙胁迫 强度越大, 物质积累越少, 植物的高生长越慢。3) 由于风沙流降低了水分利用率, 从而增加了对植物的干燥作用。 从总体来看, 沙生植物对风沙流胁迫有一定的适应响应, 实验所用灌木较草本植物有更强的抗风性。
\end{abstract}

关键词 风沙 净光合速率 $(P n)$ 水分利用率 $(W U E)$

\section{RESPONSE OF THE ECO-PHYSIOLOGICAL CHARACTERISTICS OF SOME PLANTS UNDER BLOWN SAND}

\author{
YU Yun-Jiang $^{1}{ }^{2}$ SHI Pei-Jun ${ }^{1} \quad$ LU Chun-Xia ${ }^{3}$ and LIU Jia-Qiong ${ }^{2}$ \\ (1 Institute of Resource Science, Key Laboratory of Environmental Change and Natural Disaster ,the Ministry of Education \\ of China , Beijing Normal University , Beijing 100875 , China) \\ (2 Desert Experimental Research Station at Sha Po-tou, Cold and Arid Regions Environment and Engineering Research Institute , \\ the Chinese Academy of Sciences , Lanzhou 730000 , China) \\ (3 Institute of Geographical Sciences and Natural Resources Research, the Chinese Academy of Sciences , Beijing 100101, China)
}

\begin{abstract}
Blown sand is very common in nature. In areas with frequent blown sand, especially arid and semiarid areas , blown sand not only buries highways and railways but also poses a threat to the sustainable use and development of plant resources. In the past, researchers have conducted many studies of sand-burying and wind erosion, but there are few studies on the effect of blown sand on plant eco-physiology. The nature of the influence of blown sand on the eco-physiological characteristics of plants is an important question. Though scientists have observed and studied some effects of wind on morphological characters and transpiration ratios the effects of blown sand on the net photosynthetic rate, water use efficiency etc. of plants are unknown. In this paper, based on experimental methods we showed relations between different blown sand conditions and some ecophysiological characteristics in plants , and revealed adaptability of experimental plants to blown sand by observing the change of photosynthesis and water use efficiency in plants.

In this paper, using a field wind tunnel, the effects of blown sand on the growth characteristics of some sand-fixing plants ( Eragrostis poaeoides Beauv. , Agriophyllum squarrosum , Bassia dasyphylla Kuntze , Caragana korshinskii Kom. , Artemisia ordosica Krasch, Reaumuria soongorica Maxim , Ammopiptanthus mongolicus Cheng f. , Hedysarum scoparium L. , Robinia pseudoacacia L.) were studied under different wind conditions including different wind velocities $\left(5.9,7.9,9.9,14 \mathrm{~m} \mathrm{~s}^{-1}\right.$ etc. $)$ and blowing intervals $(2 \mathrm{~d}, 4 \mathrm{~d}, 9$ d), and some eco-physiological parameters were measured. The results showed : 1) Both wind and wind-sand current made net photosynthetic ratio $(P n)$ decrease and transpiration ratio $(E)$ rise, and thus made water use efficiency (WUE) decrease. 2) The larger the wind velocity was or the shorter the blowing interval by windsand was, the larger the reduction in $P n$; the effect of wind-sand current on the above index was greater than the effect of pure wind. The more fierce the wind-sand menace to the plants was , the less the substance accumulation was , and thus the more slowly the plant grew in height ;3) Wind-sand current aggravated desiccation
\end{abstract}


of plants, due to the reduction in WUE. Meanwhile sand-fixing plants have adaptability to wind-sand current. The shrubs showed more adaptability to wind-sand current than the grasses. According to the change of WUE in experimental plants under blown sand conditions, the adaptability of experimental shrubs to blown sand ranks as follows : Reaumuria soongorica > Ammopiptanthus mongolicus > Caragana korshinskii > Artemisia ordosica $>$ Hedysarum scoparium $>$ Robinia pseudoacacia. This ranking corresponds with the order in their capability of resisting drought. (4) The affecting capability of the blown sand on different plants is different. In this experiment, the change in $P n$ and WUE for grasses is more than shrubs, and the change for Artemisia ordosica is less than for Agriophyllum squarrosum under higher velocity.

Key words Blown sand, Net photosynthetic ratio $(P n)$, Water use efficiency ( WUE)

风沙活动不仅是我国北方干旱地区一种普遍现 象, 而且在南方局部地区也有发生。在风沙流活动 频繁的地区,形成了不同形式、不同程度的风沙灾 害 既造成了沙埋铁路、公路及掩埋农田等灾害现 象, 同时也直接威胁着这些地区生物资源的可持续 利用与发展。随着全球变暖、环境旱化, 近年来我国 的沙尘暴发生频次增加, 范围和强度也在增大(史培 军等 2000)。以往关于风沙引起的沙埋、风蚀现象， 人们给予了较多的关注和研究, 但有关风沙胁迫对 植物生态生理影响的研究尚在初步探索阶段。

前人曾探索了植物群体对风速的拦阻作用，如 防护林对远方风速的重新分配( 凌裕泉, 1988)、玉米 群体密度对风速的影响(刘开昌等,2000) 等。有关 风沙对植物生态生理影响的研究, 主要集中在净风 对蒸腾作用 (潘瑞炽等, 1984) 和外形特征的影响方 面，如 Griddings (1994) 就强风对蒸腾的影响进行了 研究, R.F.道本迈尔 (1986) 注意到风使植物呈旗形 发育。上述这些研究只局限于植物的蒸腾过程以及 外部形态的变化上, 而没有关注风沙对植物物质积 累(即碳同化)和水分利用的影响。本文试图借助实 验手段，通过对不同风况下植物光合作用及水分利 用状况定量的研究, 认识各种风沙条件与植物生态 生理特征的关系，从而揭示几种常见沙生植物对风 沙流的适应能力。

\section{1 实验内容和研究方法}

实验在中国科学院寒区旱区环境与工程研究所 沙坡头实验站的野外风洞实验室里进行。

\section{1 材料和风况设计}

\subsection{1 实验材料}

A.野外植物 :a)草本植物: 小画眉草 (Eragrostis poaeoides Beauv.) 和雾冰藜 (Bassia dasyphylla Kuntze) ;b) 灌木半灌木 :柠条 ( Caragana korshinskii Kom.) 和油蓠 (Artemisia ordosica Krasch)。这两类植 物均取自沙坡头人工植被种植区，采用植物活体连 同着生的土壤，采集的土体大小为 $50 \mathrm{~cm} \times 50 \mathrm{~cm} \times$
$50 \mathrm{~cm}$ 。

B. 盆栽植物 :红砂 ( Reaumuria soongorica Maxim)、沙冬青 (Ammopiptanthus mongolicus Cheng f.)、花 棒 (Hedysarum scoparium L.) 、油蒿、柠条和刺槐 (Robinia pseudoacacia L.) ,这些植物均栽植在 $30 \mathrm{~cm}$ (直径) $\times 40 \mathrm{~cm}$ (高)的容器盆内。

\subsection{2 风况}

1)不同风速的净风和风沙流 :采用的风况分别 为 5.9、7.9、9.9、14 $\mathrm{m} \mathrm{s}^{-1}$ 的净风和风沙流。2) 相同 速度 $\left(15 \mathrm{~m} \mathrm{~s}^{-1}\right)$ 不同间隔 $(2 \mathrm{~d} 、 4 \mathrm{~d} 、 9 \mathrm{~d})$ 的风沙流。 供试植物油蒿为春季播种的盆栽植物, 实验时油蒿 平均高 $56 \mathrm{~cm}$;沙米 (Agriophyllum squarrosum) 是 6 月 份从野外移栽的 到实验时平均高 $33 \mathrm{~cm}$ 。实验采用 生长状况相对一致的苗木进行, 对以上两植物均采 用常规管理。3) 不同风速。为了解不同风速风沙 流对植物高生长的影响，除了采用不同间隔的处理 外, 还用较大风速 $\left(10 、 15 、 20 \mathrm{~m} \mathrm{~s}^{-1}\right)$ 的风沙流。供 试油蒿、沙米与不同间隔时的生长状况相同。以上 每种风况对植物吹袭 1 次, 做 3 个重复。吹袭时间 持续 $45 \mathrm{~min}$ 。

\subsection{3 测定项目及方法}

净光合速率 $(P n)$ 和蒸腾速率 $(E)$ 采用 Li-6200 便携式气体分析系统 (Li-cor lincoln, NE. USA)测定 ; 计算 $P n 、 E$ 所需的叶面积参数使用 $\mathrm{Li}-3000 \mathrm{~A}$ 便携 式叶面积仪 (Li-corlincoln ,NE, USA)测定。

测定设计 :分别在风吹前后即时测定，所测定的 叶片为冠层中上部新枝上的成熟叶片, 每次测定 3 个叶子, 在每个叶子上重复测定 3 次。 $W U E=P n /$ E。

\subsection{4 数据分析方法}

试验数据的统计分析采用方差分析法。

\section{2 结果与分析}

\section{1 不同风速的净风和风沙流对 $P n 、 E 、 W U E$ 的影} 响 
$\mathrm{m} \mathrm{s}^{-1}$ )的吹袭下 植物的 $P n$ 均降低, $E$ 均有上升。

2.1.1Pn、E 的变化

在净风和风沙流胁迫下, 随着风速增大, 不论灌 木半灌木 (油蒿和柠条) 还是草本植物 (小画眉草和 雾冰藜)的净光合速率 $(P n)$ 均显著降低(图 1)，且风 速越大、降幅越大 ; 草本植物比灌木植物的净光合速 率降幅大，如在 $14 \mathrm{~m} \mathrm{~s}^{-1}$ 的风速下，净风可使草本植 物 $P n$ 降低 $34.42 \%$, 灌木仅降低 $27.54 \%$ 。蒸腾速 率 $(E)$ 与 $P n$ 的变化相反, 随风速增大而增大 (图 2)。

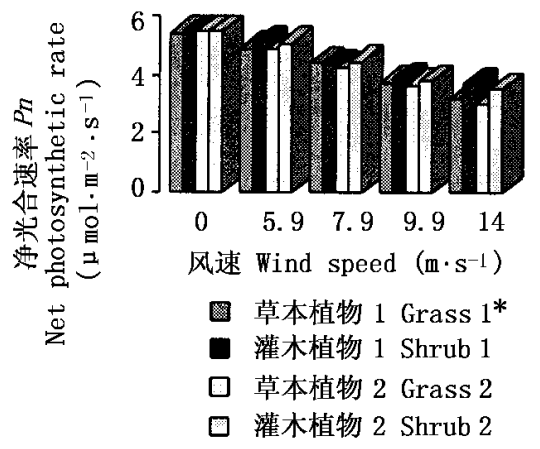

图 1 风、风沙流下, 不同植物 $P n$ 的变化

Fig. $1 \quad P n$ change of different species under wind and wind-sand current * 植物后数字表示不同的条件 The number behind plants means: 1. 净风 Wind 2. 风沙流 Wind-sand current

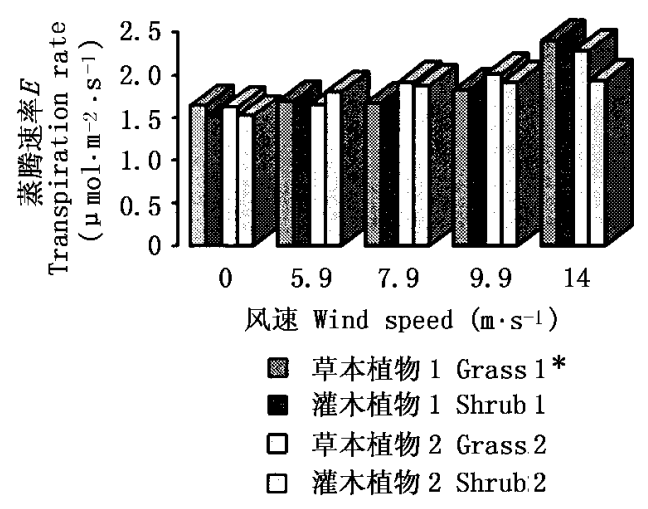

图 2 风、风沙流下, 不同植物 $E$ 的变化

Fig.2 Transpiration $E$ change of different species under wind and wind-sand current

* 同图 1 See Fig. 1

\subsection{2 水分利用效率 $(W U E)$ 的变化}

由于 $P n$ 随着风速增加而降低, $E$ 随着风速增 加而减少，因此水分利用效率( WUE) 随风速的增大 而降低(图 3)。在 $14 \mathrm{~m} \mathrm{~s}^{-1}$ 风沙流下, 草本植物的 WUE 降低了 $60.24 \%$ 。风沙流对盆栽植物吹袭后， 几种植物有不同的降幅, WUE 降幅最大的为刺槐、 花棒, 分别为吹前 WUE 的 $18 \% 、 39 \%$ (表 1)。

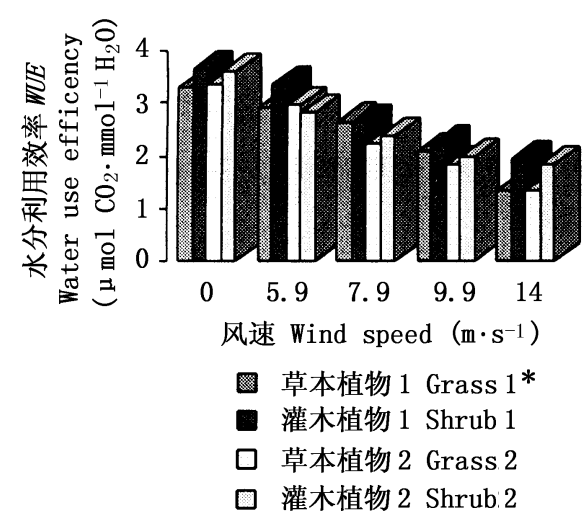

图 3 净风、风沙流条件下, 不同植物水分利用率的变化 Fig.3 WUE change of different species under wind and wind-sand current * 同图 1 See Fig. 1

表 16 种植物在风沙流吹袭下, WUE 的相对变化值

Table 1 Relative change value of WUE of six plants

\begin{tabular}{llllll}
\hline \multicolumn{1}{c}{ 植物种 } & \multicolumn{5}{c}{ 风速 Wind speed $\left(\mathrm{m} \mathrm{s}^{-1}\right)$} \\
\cline { 2 - 7 } \multicolumn{1}{c}{ Species } & 0 & 5.9 & 7.9 & 9.9 & 14 \\
\hline 油蒿 Artemisia ordosica & 1 & 0.85 & 0.65 & 0.62 & 0.48 \\
花棒 Hedysarum scoparium & 1 & 0.78 & 0.60 & 0.56 & 0.39 \\
柠条 Caragana korshinskii & 1 & 0.90 & 0.75 & 0.68 & 0.56 \\
沙冬青 Ammopiptanthus mongolicus & 1 & 0.88 & 0.81 & 0.79 & 0.58 \\
红砂 Reaumuria soongorica & 1 & 0.85 & 0.77 & 0.70 & 0.67 \\
刺槐 Robinia pseudo-acacia & 1 & 0.90 & 0.69 & 0.31 & 0.18 \\
\hline
\end{tabular}

表中数字是以 $0 \mathrm{~m} \mathrm{~s}^{-1}$ 的 WUE 为 1 时的相对值 The numbers in the table are the relative value when the value of $W U E$ is in $0 \mathrm{~m} \mathrm{~s}^{-1}$ wind speed.

\subsection{3 净风和风沙流影响的不同特点}

风沙流对 $P n$ 的影响较净风大，同在 $14 \mathrm{~m} \mathrm{~s}^{-1}$ 风速下,风沙流使草本植物的 $P n$ 降低 $40.7 \%$,大于 净风的降低幅度 $(35.88 \%)$ 。

\section{2 不同间隔风沙流对 $P n 、 E 、 W U E$ 的影响}

\subsection{1 $P n 、 E$ 的变化}

风沙流的间隔愈小、Pn 降低愈大, 且彼此的降 幅差异明显。 $2 \mathrm{~d}$ 吹 1 次、 $4 \mathrm{~d}$ 吹 1 次、 $9 \mathrm{~d}$ 吹 1 次的 处理分别使油蒿 $P n$ 降低 $20.13 \%, 11.76 \%, 7.72 \%$ ， 远比其对照的变化大(表 2)。沙米在上述条件下的 降幅比油蒿大, 分别降低了 $38.97 \%, 21.57 \%, 14$. $72 \%$ (表 3)。风沙流的不同吹袭间隔对蒸腾速率的 影响并没有明显的差异。

\section{2 .2 水分利用效率}

试验结果表明，不同吹袭时间间隔的风沙流均 能显著影响油蒿和沙米的水分利用效率, 使其明显 下降(表 2 , 表 3) ; 并且不同吹袭时间间隔模式对植 物水分利用效率的影响有显著的差异, 间隔愈短, 水 分利用效率降幅愈大。油蒿、沙米在 $2 \mathrm{~d}$ 吹 1 次的 处理下分别降低 $37.28 \% 、 55.33 \%$, 远大于 $9 \mathrm{~d}$ 吹 1 次的降幅(分别为 $22.00 \% 、 34.84 \%$ )。 
表 2 不同间隔下, 油蓠 (Artemisia ordosica) 的 Pn、E、WUE 变化

Table 2 Change of Artemisia ordosica for $P n, E$,WUE under different intervals *

\begin{tabular}{|c|c|c|c|c|c|c|c|c|}
\hline \multirow{3}{*}{ 处理 Treatment } & \multicolumn{8}{|c|}{ 参数 Parameters } \\
\hline & \multicolumn{3}{|c|}{$P n\left(\mu \mathrm{mol} \mathrm{m} \mathrm{m}^{-2} \mathrm{~s}^{-1}\right)$} & \multicolumn{2}{|c|}{$E\left(\mathrm{mmol} \mathrm{m} \mathrm{m}^{-2} \mathrm{~s}^{-1}\right)$} & \multicolumn{3}{|c|}{ WUE $\left(\mu \mathrm{mol} \mathrm{CO}_{2} \mathrm{mmol}^{-1} \mathrm{H}_{2} \mathrm{O}\right)$} \\
\hline & A & $\mathrm{B}$ & 变化率 Change rate (\%) & A & B & A & B & 变化率 Change rate (\%) \\
\hline $\mathrm{t}_{1}(2 \mathrm{~d}$ 一吹 $)$ & 6.36 & 5.08 & $-20.13^{a}$ & 1.49 & 1.81 & 4.48 & 2.81 & $-37.28^{\mathrm{a}}$ \\
\hline $\mathrm{t}_{2}(4 \mathrm{~d}$ 一吹 $)$ & 6.89 & 6.08 & $-11.76^{b}$ & 1.46 & 1.73 & 4.72 & 3.48 & $-26.27^{b}$ \\
\hline $\mathrm{t}_{3}(9 \mathrm{~d}$ 一吹 $)$ & 6.74 & 6.22 & $-7.72^{\mathrm{b}}$ & 1.53 & 1.81 & 4.41 & 3.44 & $-22.00^{\mathrm{b}}$ \\
\hline $\mathrm{C}_{1}$ (对照 1) & 6.15 & 6.09 & $-0.98^{\mathrm{c}}$ & 1.42 & 1.39 & 4.33 & 4.38 & $1.15^{\mathrm{c}}$ \\
\hline $\mathrm{C}_{2}$ (对照 2) & 6.54 & 6.62 & $1.22^{\mathrm{c}}$ & 1.36 & 1.46 & 4.81 & 4.53 & $-5.82^{\mathrm{c}}$ \\
\hline $\mathrm{C}_{3}$ (对照 3 ) & 6.26 & 6.22 & $-0.64^{\mathrm{c}}$ & 1.38 & 1.43 & 4.54 & 4.35 & $-4.19^{\mathrm{c}}$ \\
\hline
\end{tabular}

A、B 分别表示吹前、吹后 $\quad \mathrm{a} 、 \mathrm{~b} 、 \mathrm{c}$ 间有显著差异, $p=0.05$ 变化率 :负值表示减少,正值表示增加 A stands for the parameter before wind blown , B stands for that after wind blown There is a remarkable difference among a , b , c , $p=0.05$ Negative sign for change rate means decrease and the positive sign means increase

表 3 不同时间间隔下沙米的 $P \boldsymbol{P} 、 \boldsymbol{E} 、 \boldsymbol{W U E}$ 的变化

Table 3 Change of Agriophyllum squarrosum for $P n, E$,WUE under different intervals

\begin{tabular}{|c|c|c|c|c|c|c|c|c|}
\hline \multirow{3}{*}{ 处理 Treatment } & \multicolumn{8}{|c|}{ 参数 Parameters } \\
\hline & \multicolumn{3}{|c|}{ 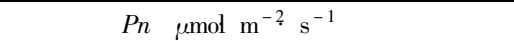 } & \multicolumn{2}{|c|}{$E\left(\mathrm{mmol} \mathrm{m} \mathrm{m}^{-2} \mathrm{~s}^{-1}\right)$} & \multicolumn{3}{|c|}{ WUE $\left(\mu \mathrm{mol} \mathrm{CO}_{2} \mathrm{mmol}^{-1} \mathrm{H}_{2} \mathrm{O}\right)$} \\
\hline & A & B & 变化率 Change rate (\%) & A & $\mathrm{B}$ & A & $\mathrm{B}$ & 变化率 Change rate $(\%)$ \\
\hline $\mathrm{t}_{1}(2 \mathrm{~d}$ 一吹 $)$ & 6.21 & $3.79^{*}$ & $-38.97^{\mathrm{a}}$ & 1.38 & 1.89 & 4.50 & 2.01 & $-55.33^{\mathrm{a}}$ \\
\hline $\mathrm{t}_{2}$ (4 d 一吹) & 6.86 & $5.38^{*}$ & $-21.57^{\mathrm{b}}$ & 1.32 & 1.78 & 5.20 & 2.82 & $-45.77^{b}$ \\
\hline $\mathrm{t}_{3}(9 \mathrm{~d}$ 一吹 $)$ & 6.93 & $5.91^{*}$ & $-14.72^{\mathrm{c}}$ & 1.42 & 1.86 & 4.88 & 3.18 & $-34.84^{\mathrm{c}}$ \\
\hline $\mathrm{C}_{1}$ (对照 1) & 6.31 & 6.28 & $-0.63^{\mathrm{d}}$ & 1.41 & 1.38 & 4.48 & 4.55 & $1.43^{\mathrm{d}}$ \\
\hline $\mathrm{C}_{2}$ (对照 2) & 6.72 & 6.71 & $-0.15^{\mathrm{d}}$ & 1.45 & 1.36 & 4.63 & 4.93 & $6.48^{\mathrm{d}}$ \\
\hline $\mathrm{C}_{3}$ (对照 3) & 6.81 & 6.66 & $-2.20^{\mathrm{d}}$ & 1.39 & 1.31 & 4.90 & 5.08 & $3.67^{\mathrm{d}}$ \\
\hline
\end{tabular}

同表 1 See Table 1

表 4 不同条件风沙流对油蒿、沙米高生长 $(\mathrm{cm})$ 的影响

Table 4 Effect of different wind-sand current on height growth of Artemisia ordosica and Agriophyllum squarrosum

\begin{tabular}{|c|c|c|c|c|c|c|c|c|c|}
\hline \multirow[b]{2}{*}{ 植物种 Species } & \multicolumn{4}{|c|}{ 风速 Wind speed $\left(\mathrm{m} \mathrm{s}^{-1}\right)$} & \multirow[b]{2}{*}{ 植物种 Species } & \multicolumn{4}{|c|}{ 间隔 Interval (d) } \\
\hline & 10 & 15 & 20 & $\begin{array}{c}\text { 对照 } \\
\text { Control }\end{array}$ & & 2 & 4 & 9 & $\begin{array}{c}\text { 对照 } \\
\text { Control }\end{array}$ \\
\hline 油蒿 A. ordosica & 3.52 & 2.48 & 1.81 & 4.87 & 油蒿 A. ordosica & 1.98 & 2.48 & 2.66 & 4.94 \\
\hline 沙米 A. squarrosum & 3.16 & 2.26 & 1.56 & 4.68 & 沙米 A. squarrosum & 1.72 & 2.26 & 2.48 & 4.86 \\
\hline
\end{tabular}
times)

\section{3 不同风况风沙流对油蓄、沙米高生长的影响}

为了了解不同风速和不同吹袭时间间隔的风沙 流对植物高生长的影响，分别用较大的不同风速 $\left(10 、 15 、 20 \mathrm{~m} \mathrm{~s}^{-1}\right)$ 、不同吹袭时间间隔 $(2 、 4 、 9 \mathrm{~d})$ 的 风沙流对油蒿、沙米进行吹袭实验。从一个月的生 长量 (表 4) 可知, 风速愈大, 高生长愈慢, 如 $10 \mathrm{~m} \mathrm{~s}^{-1} 、 15 \mathrm{~m} \mathrm{~s}^{-1} 、 20 \mathrm{~m} \mathrm{~s}^{-1}$ 下油蒿的高生长分别 为对照的 $72.28 \% 、 50.92 \% 、 37.17 \%$; 风沙流间隔愈 短, 高生长受到制约也愈大 $2 \mathrm{~d}, \mathrm{Ad}, 9 \mathrm{~d} 3$ 种间隔处 理的高生长分别是对照的 $40.08 \% 、 50.20 \% 、 53$. $85 \%$ 。

\section{3 讨 论}

\section{1 净风和风沙流对植物生长影响的共同点} 由实验结果可知, 净风和风沙流均使植物生长
变慢。从本研究还可知道, 油葶、沙米高生长随风况 的变化与植物净光合速率随风况变化相一致 (表 2 , 表 3 表 4)，这可能说明风和风沙流通过限制植物光 合作用这一生理途径, 阻遏植物碳同化能力, 降低了 植物的物质积累, 进而使植物生长受到影响。蒸腾 作用是随环境因子的变化而迅速变化的生理生态过 程(王铸豪，1986)。实验表明，风和风沙流能促进蒸 腾, 这是因为它们能将气孔外边的水蒸气吹走, 补充 一些相对湿度较低的空气，使扩散层变薄或消失，外 部扩散阻力减小, 从而蒸腾加快。风和风沙流胁迫 导致蒸腾和光合速率的变化当然也会引起水分利用 效率 WUE 的变化。随着风沙流胁迫的加大, 光合 速率 $P n$ 减小 蒸腾速率 $E$ 增大 结果导致水分利用 效率 WUE 减小; $W U E$ 的减少说明了风和风沙流间 接地加剧了植物的干旱胁迫。 


\section{2 不同风况对植物生长影响的差别}

\subsection{1 净风和风沙流}

风是一个很重要的环境因子，它对植物生长的 影响有矮化、畸形等作用，而风沙流则增加了擦伤、 打击和沙的蚀积等作用。风沙流是一种气固两相 流，它是起沙风通过对地面侵蚀，使不饱和气流携带 沙子而形成的混合流, 它具有净风所没有的特征(朱 震达等，1986），在吹袭植物时风沙流中的沙子直接 打击到植物体上, 使植物外部形态和生理结构都受 到损伤。就本文的实验风速来看 较低风速 (5.9.7. 9、9.9 $\mathrm{m} \mathrm{s}^{-1}$ )下的净风和风沙流对植物 $P n$ 的影响， 并无明显的差异, 但当风速增为 $14 \mathrm{~m} \mathrm{~s}^{-1}$ 时，风沙流 使 $P n$ 的降幅就明显大于净风。对于形态特征的影 响，净风和风沙流的差异则在较低风速时就出现。 当 $7.9 \mathrm{~m} \mathrm{~s}^{-1}$ 净风吹击时, 油蒿倾斜、全株抖动，叶子 卷曲 草本植物则从根部发生剧烈颤动 根部土壤受 到吹蚀。而当 $7.9 \mathrm{~m} \mathrm{~s}^{-1}$ 的风沙流吹袭时, 除了出现 净风吹袭时的反应外，还增加了以下症状 油蕎的部 分叶子被打掉, 局部有断梢现象, 形成了较大的损 害 ;草本植物则背后有积沙出现。

\section{2 .2 速度和间隔}

从本研究的实验结果可以看出, 凤沙的速度和 吹袭的时间间隔是影响植物生长的另外两个关键因 子。风和风沙流可能制约着植物的光合作用, 植物 的碳同化可能与风沙流的胁迫强度密切相关, 风速 大、吹风间隔短的风沙流使光合速率降低最大, 造成 植物的物质积累与生长缓慢 (图 1 , 表 4)。高频率的 胁迫往往在植物的生态生理状况还未完全恢复时就 又给予吹击，从而对植物生态生理的影响更大。

\subsection{3 灌、草植物对风和风沙流的适应性}

在低风速处理组 $\left(5.9 、 7.9 、 9.9 、 14 \mathrm{~m} \mathrm{~s}^{-1}\right)$ 中， 在同样风况下, 灌木半灌木 (油蒿、柠条) $P n$ 的降幅 比草本植物 (雾冰藜、小画眉草)小; 在较高风速处理 组 $\left(10 、 15 、 20 \mathrm{~m} \mathrm{~s}^{-1}\right)$ 中, 半灌木油蓠 $P n$ 的降幅也比 草本植物沙米的小(表 2 , 表 3 )。由此可见，风沙流 对供试灌木半灌木的光合作用影响要比对草本植物 的影响小。此外, 在同样速度的风沙流吹袭下, 油蒿 水分利用效率降幅也小于沙米，譬如，同在两天吹 1 次风沙流的处理下, 油蒿的水分利用效率降低 37. $28 \%$,而沙米的水分利用效率则降低了 $55.33 \%$ (表 2 ,表 3 )。

沙漠生态系统是一个脆弱的生态系统, 灌木是 该生态系统中最重要、最活跃的成分，对稳定与保护 生态环境, 支持草地畜牧业经济具有极其重要的地
位和意义(张新时,1994)。本实验选用的灌木均为 典型的旱生植物。其中油蒿和柠条是许多沙地的代 表性灌木植物，它们对沙漠环境有较好的适应性(张 强,1983; 丘明新,1984;沈渭寿,1986)。特别是油 蒿, 被认为是腾格里沙漠东南缘和毛乌素沙地最优 良的固沙植物（赵兴梁,1988)。水分利用效率是沙 生植物适应旱生环境的一个重要指标。风沙流胁迫 下，不同沙生植物的生态生理反应是不同的，通过对 油蓠、柠条、红砂、花棒、刺槐、沙冬青的风沙流吹袭 实验, 可以看出风沙流使它们的水分利用效率均有 降低 (表 1), 但降低程度有明显的差异。以 14 $\mathrm{m} \mathrm{s}^{-1}$ 风沙流条件下 WUE 降幅大小为依据来排列， 则几种植物水分利用效率降幅从小到大依次为: 红 砂、沙冬青、柠条、油蒿、花棒、刺槐(表 1)。若以降 幅少作为适应性强的依据，则这几种植物适应风沙 流能力从大到小的排列也应为 红砂 $>$ 沙冬青 $>$ 柠 条 $>$ 油蒿 $>$ 花棒 $>$ 刺槐。这个顺序与它们在旱生环 境中的实际抗旱能力相符。红砂、沙冬青的抗风沙 能力强, 但生长极缓慢, 生物量低, 短期内难以达到 固沙的要求, 因此在流沙治理阶段不宜采用。虽然 风沙流胁迫下 柠条的水分利用效率较油蒿大, 但油 蒿的净光合速率比柠条高, 能以其肉质化程度较高 的叶进行光合作用, 并具有较低的胞间 $\mathrm{CO}_{2}$ 浓度 (张 利平等,1998)，此外，油蒿还具有种子源丰富，繁殖 容易等特点(王刚等,1995)，这些使得油蒿在旱生环 境中比柠条有更强的适应能力。

\section{参 考 文 献}

Griddings, L. A. 1914. Evanspiration of silphium Laciniatam L. Plant World, 35:937 942 .

Ling, Y. Q. (凌裕泉). 1988. Principle of physics of blown sand on railway sand-controlling - for example, Baotou-Lanzhou Railway. In: Desert Experimental Research Station at Shapotou, the Chinese Academy of Sciences (中国科学院沙坡头沙漠试验站) ed. Lanzhou: research on fluid sand at Shapotou area at the southeastern edge of Tengger Desert II . Yinchuan: Ningxia People's Publishing House. 297 308. (in Chinese)

Liu, K.C. (刘开昌), X. Q. Zhang (张秀清), Q.C. Wang (王 庆成), C.Y. Wang (王春英) \& A.Q. Li (李爱芹). 2000. Effect of plant of density on microclimate in canopy of maize. Acta Phytoecologica Sinica (植物生态学报), 24:489 493. (in Chinese with English abstract)

Pan, R.Z. (潘瑞炽) \& Y.D. Dong(董愚得). 1984. Plant physiology. Beijing: China Higher Education Press. 17 24, 132 . (in Chinese)

Qiu, M. X. (丘明新). 1984. Research on relation between Artemisia ordosica community and controlling-sand afforestationdiscussion on probability of building artificial vegetation by means of the community. Acta Botanica Boreali-Occidentalia Sinica(西 北植物学报), 4:29 39. (in Chinese with English abstract) 
R.F. 道本迈尔. 1986. (translated by Qu, Z.X. (曲仲湘)). Plant and environment. Beijing: Science Press. $60 \sim 64$. (in Chinese)

Shen, W.S. (沈渭寿). 1986. The station of Artemisia ordosica in vegetation succession of Shapotou area. Journal of Desert Research(中国沙漠), 6 (4) : 13 21. (in Chinese with English abstract)

Shi, P. J. (史培军), P. Yan (严平) \& S. Y. Gao(高尚玉). 2000. The duststorm disaster in China and its research progress. Journal of Natural Disasters(自然灾害学报),9(3): 71 77. (in Chinese with English abstract)

Wang, G. (王刚) \& X.G. Liang(梁学功). 1995. The seed bank dynamic of sand dune of artificial vegetation in Shapotou area. Acta Botanica Sinica (植物学报), 37:231 23. (in Chinese with English abstract)

Wang, Z.H. (王铸豪) . 1986. Plant and environment. Beijing: Science Press. $60 \sim 64$. (in Chinese)

Zhang, L.P. (张利平), X.P. Wang (王新平) \& L.C. Liu(刘立 超). 1998. Study on gas exchange characteristics of main con- structive plants A. ordosica and C. korshinskii in Shapotou Region. Acta Phytoecologica Sinica (植物生态学报), 18: 133 137. (in Chinese with English abstract)

Zhang, Q. (张强). 1983. Study on renewing of Artemisia ordosica community. Chinese Journal of Ecology (生态学杂志), 2 (2) : 14 $\sim 19$. (in Chinese)

Zhang, X.S. (张新时). 1994. The ecological background of the $\mathrm{Mu}$ Us sandland and the principles and optimal models for grassland management. Acta Phytoecologica Sinica(植物生态学报), 18: $1 \sim 16$. (in Chinese with English abstract)

Zhao, X.L. (赵兴梁). 1988. Research on problem of controllingsand of vegetation revearch on fluid sand at Shapotou area at the southeastern edge of Tengger Desert (2). Yinchuan: Ningxia People's Publishing House. $27 \sim 57$. (in Chinese)

Zhu, Z.D. (朱震达), S. Liu (刘恕) \& X.M. Di (邸醒民). 1986. Development and harmess of land desertification in China. Mem. of Institute of Desert Academica Sinica Lanzhou China (中 国科学院兰州沙漠所集刊), 35. (in Chinese)

责任编委 : 傅伯杰 责任编辑 :姜联合 